\title{
ENUMERATION OF PLANE PARTITIONS BY DESCENTS
}

\author{
DAMIR YELIUSSIZOV
}

\begin{abstract}
We study certain bijection between plane partitions and $\mathbb{N}$-matrices. As applications, we prove a Cauchy-type identity for generalized dual Grothendieck polynomials. We introduce two statistics on plane partitions, whose generating functions are similar to classical MacMahon's formulas; one of these statistics is equidistributed with the usual volume. We also show natural connections with the longest increasing subsequences of words.
\end{abstract}

\section{INTRODUCTION}

One of the most successful bijections in combinatorics is the Robinson-Schensted-Knuth (RSK) correspondence. It has many important properties and applications, see [Sta99, Ch. 7 \& notes]. In particular, the RSK gives a bijection between $\mathbb{N}$-matrices and plane partitions.

In this paper, we study a different (yet somewhat analogous) bijection between these sets. A map from plane partitions to matrices is quite transparent; it is described by recording the so-called descent level sets (or can be viewed by projecting corners in $\mathbb{R}^{3}$ presentation).

This approach gives a number of interesting consequences. We obtain the following results:

- A multivariate identity via certain descent statistics of plane partitions. The identity becomes a source of several subsequent formulas.

- Generating functions for two volume-type statistics. We call them the up-hook and corner volumes. The formulas are analogous to classical MacMahon's identities. Interestingly, the up-hook volume is equidistributed with the usual volume of plane partitions.

- A Cauchy-type identity for generalized dual symmetric Grothendieck polynomials which are $K$-theoretic inhomogeneous deformations of Schur functions.

- A Frobenius-type identity for strict tableaux which are generalizations of the standard Young tableaux (SYT). We show that these objects are naturally related to the longest increasing subsequences of words.

- Monotonicity and bounds for certain descent enumeration functions of plane partitions.

\section{Preliminaries}

2.1. Partitions. A partition is a sequence $\lambda=\left(\lambda_{1}, \ldots, \lambda_{\ell}\right)$ of positive integers $\lambda_{1} \geq \cdots \geq \lambda_{\ell}$, where $\ell(\lambda)=\ell$ is the length of $\lambda$. Every partition $\lambda$ can be represented as the Young diagram $\left\{(i, j): i \in[1, \ell], j \in\left[1, \lambda_{i}\right],(i, j) \in \mathbb{N}^{2}\right\}$.

2.2. Plane partitions. A plane partition $\pi=\left(\pi_{i j}\right)_{i, j \geq 1}$ is a two-dimensional array of nonnegative integers with finitely many nonzero entries such that

$$
\pi_{i j} \geq \pi_{i+1 j}, \quad \pi_{i j} \geq \pi_{i j+1}, \quad \text { for all } i, j \geq 1 .
$$

We denote by $|\pi|:=\sum_{i, j} \pi_{i j}$ the volume (or size, or total weight) of $\pi$. 


\begin{tabular}{|l|l|l|}
\hline 4 & 3 & 2 \\
\hline 3 & 3 & 1 \\
\hline
\end{tabular}

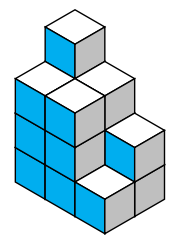

Figure 1. A plane partition $\pi \in \operatorname{PP}(3,2,4)$ with $|\pi|=16, \operatorname{sh}(\pi)=(3,3)$, and its boxed presentation.

By default we ignore zero entries of plane partitions. The shape of $\pi$ denoted by $\operatorname{sh}(\pi)$ is the partition whose Young diagram is $\left\{(i, j): \pi_{i j}>0\right\}$. It is useful to view plane partitions as a pile of cubes in $\mathbb{R}^{3}$ as in Fig. 1 . They can be bounded by a box. Let $\operatorname{PP}(k, n, m)$ be the set of plane partitions that fit in the box $k \times n \times m$, i.e. the length of the first row is at most $k$, the length of the first column is at most $n$, and the first entry is at most $m$. Let also $\operatorname{PP}^{\prime}(k, n, m)$ be the set of plane partitions whose base shape is exactly $k \times n$, i.e. the first row length is $k$, the first column length is $n$, and the first entry is still at most $m$.

We have classical MacMahon's formula [Mac16]:

$$
\sum_{\pi \in \operatorname{PP}(k, n, m)} q^{|\pi|}=\prod_{i=1}^{k} \prod_{j=1}^{n} \prod_{\ell=1}^{m} \frac{1-q^{i+j+\ell-1}}{1-q^{i+j+\ell-2}}
$$

which for $k, n, m \rightarrow \infty$ gives the generating function

$$
\sum_{\pi} q^{|\pi|}=\prod_{n=1}^{\infty} \frac{1}{\left(1-q^{n}\right)^{n}} .
$$

For $q \rightarrow 1$, we have MacMahon's famous theorem on boxed plane partitions

$$
|\mathrm{PP}(k, n, m)|=\prod_{i=1}^{k} \prod_{j=1}^{n} \prod_{\ell=1}^{m} \frac{i+j+\ell-1}{i+j+\ell-2}
$$

We refer to [Sta99, Ch.7] for enumerative aspects of plane partitions and to an excellent more recent survey [Kra16].

2.3. Schur polynomials. The Schur polynomials $\left\{s_{\lambda}\right\}$ can be defined as follows:

$$
s_{\lambda}\left(x_{1}, \ldots, x_{m}\right):=\sum_{\pi \in \operatorname{SPP}(\lambda)} \prod_{i=1}^{m} x_{i}^{a_{i}(\pi)},
$$

where $\operatorname{SPP}(\lambda)$ is the set of column-strict plane partitions of shape $\lambda$ (i.e. $\pi_{i j}>\pi_{i+1 j}$ ) with the largest entry at most $m$, and $a_{i}(\pi)$ is the number of entries $i$ in $\pi$.

The following identities are known:

$$
\sum_{\pi \in \operatorname{PP}(k, n, m)} q^{|\pi|}=q^{-n\left(\begin{array}{c}
k+1 \\
2
\end{array}\right)} s_{\rho}\left(q, q^{2}, \ldots, q^{n+m}\right),
$$

where $\rho=(k, \ldots, k):=\left(k^{n}\right)$ is a rectangular partition; for $q=1$ this gives

$$
|\mathrm{PP}(k, n, m)|=s_{\rho}\left(1^{n+m}\right),
$$

where $\left(1^{n}\right)=(1, \ldots, 1)$ repeated $n$ times. 
2.4. Dual Grothendieck polynomials. These functions are certain $K$-theoretic deformations of Schur polynomials. They were first explicitly described and studied in [LP07].

Definition 2.1. The dual symmetric Grothendieck polynomials $\left\{g_{\lambda}\right\}$ can be defined as follows

$$
g_{\lambda}\left(x_{1}, \ldots, x_{m}\right):=\sum_{\operatorname{sh}(\pi)=\lambda} \prod_{i=1}^{m} x_{i}^{c_{i}(\pi)}
$$

where the sum runs over plane partitions $\pi$ (with largest entry at most $n$ ) of shape $\lambda$, and $c_{i}(\pi)$ is the number of columns of $\pi$ containing $i$.

The following properties are well known [LP07]: $g_{\lambda}$ is a symmetric polynomial, $g_{\lambda}=s_{\lambda}+$ lower degree terms, and hence $\left\{g_{\lambda}\right\}$ forms a basis of the ring $\Lambda$ of symmetric functions. More properties of these functions can be found in [LP07, Yel17, Yel19].

From the definition it is not difficult to obtain the following formulas

$$
|\mathrm{PP}(k, n, m)|=g_{\rho}\left(1^{m+1}\right), \quad\left|\mathrm{PP}^{\prime}(k, n, m)\right|=g_{\rho}\left(1^{m}\right),
$$

where again $\rho=\left(k^{n}\right)$. They are a special case of more general identities that we prove in this paper, e.g. we will prove formulas similar to (4).

\section{A bijection Between plane PaRtitions AND N-MATRices}

An $\mathbb{N}$-matrix is a matrix of nonnegative integers with only finitely many nonzero elements. Define the map $\Phi:\{$ plane partitions $\} \rightarrow\{\mathbb{N}$-matrices $\}$ as follows. Given a plane partition $\pi$, define the descent level sets

$$
D_{i \ell}:=\left\{j: \pi_{i j}=\ell>\pi_{i+1 j}\right\},
$$

i.e. $D_{i \ell}$ is the set of column indices of the entry $\ell$ in the $i$ th row of $\pi$ that are strictly larger than the entry below. Let $d_{i \ell}:=\left|D_{i \ell}\right|$ and define the matrix $D:=\left(d_{i \ell}\right)_{i, \ell \geq 1}$. Set

$$
\Phi(\pi)=D .
$$

Example 3.1. When $\pi$ is the plane partition shown below,

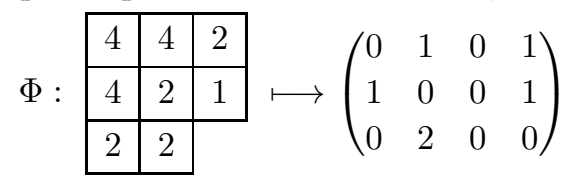

Alternatively, we can view the map $\Phi$ geometrically: present $\pi$ as a pile of cubes in $\mathbb{R}^{3}$; mark the corners $\checkmark$ on the surface of $\pi$ with $\bullet$ as in Fig. 2. Then $d_{i \ell}$ is the number of marks with the coordinates $x=i$ and $z=\ell$.

Theorem 3.2. The map $\Phi$ defines a bijection between the set $\operatorname{PP}(\infty, n, m)$ of plane partitions with at most $n$ rows and entries not exceeding $m$ and $\mathbb{N}$-matrices with $n$ rows and $m$ columns.

Proof. It is obvious that for $\pi \in \operatorname{PP}(\infty, n, m)$, the map $\Phi$ produces an $n \times m \mathbb{N}$-matrix.

Let us describe the inverse map $\Phi^{-1}$. Given an $n \times m \mathbb{N}$-matrix $D$, we show how to uniquely reconstruct a plane partition $\pi \in \operatorname{PP}(\infty, n, m)$ such that $\Phi(\pi)=D$. We will build $\pi$ sequentially by scanning the columns of $D$ starting from the last one. 


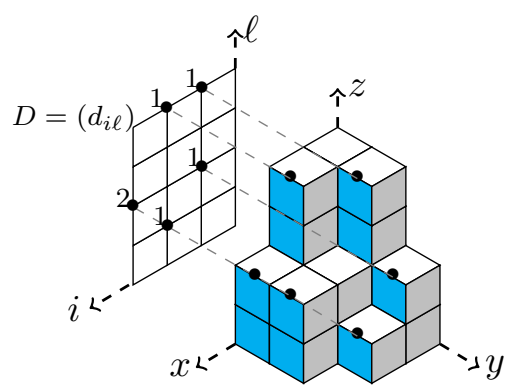

Figure 2. A geometric view of the map $\Phi$.

Let us first show how to add element $\ell$ in $i$-th row of some plane partition $\pi^{\prime}$. To do so, find the first available column of $\pi^{\prime}$ whose length is less than $i$, then add the elements $\ell$ in this column so that its length becomes $i$. For example, suppose $\pi^{\prime}$ already had the following shape and we want to add $\ell$ in the 3rd row:

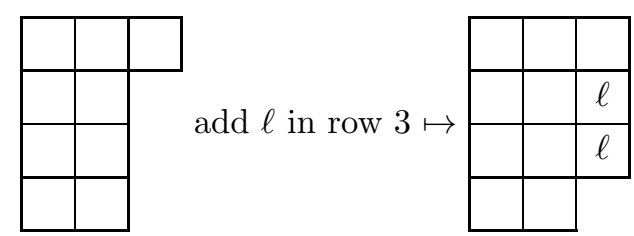

Let us initially set $\pi=\varnothing$. For each $\ell=m, m-1, \ldots, 1$ consider the $\ell$-th column $\left(d_{1 \ell}, \ldots, d_{n \ell}\right)^{T}$ of $D$. For each $i=n, n-1, \ldots, 1$ add $\ell$ in $i$-th row of $\pi$ exactly $d_{i \ell}$ times. It is easy to see that after this procedure, the resulting $\pi$ is actually a plane partition with at most $n$ rows, the entries not exceeding $m$, and that $\pi$ has the desired property, $\Phi(\pi)=D$.

Example 3.3. Let us see how $\Phi^{-1}$ applies to the matrix from Example 3.1. We have:

$$
D=\left(\begin{array}{llll}
0 & 1 & 0 & 1 \\
1 & 0 & 0 & 1 \\
0 & 2 & 0 & 0
\end{array}\right)
$$

and hence $\pi$ is constructed as follows:

add column 4 of $D: \quad$ add 4 in row 2: \begin{tabular}{|l|}
\hline \\
\hline 4
\end{tabular} add 4 in row 1: \begin{tabular}{|l|l|}
\hline 4 & 4 \\
\hline 4 &
\end{tabular}

add column 3 of $D$ : no addition

add column 2 of $D$ : add 2 in row 3 two times:

\begin{tabular}{|l|l|}
\hline 4 & 4 \\
\hline 4 & 2 \\
\hline 2 & 2 \\
\hline
\end{tabular}

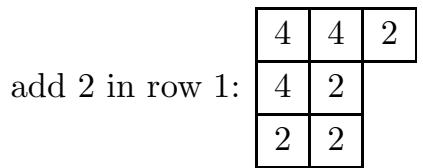

add column 1 of $D: \quad$ add 1 in row 2: \begin{tabular}{|l|l|l|}
\hline 4 & 4 & 2 \\
\hline 4 & 2 & 1 \\
\hline 2 & 2 & \multicolumn{1}{|l}{}
\end{tabular}$=\pi$

Let us point out a property of the bijection $\Phi$ that we showed in [Yel19a] for probabilistic applications. The length of the first row of $\pi$ is equal to the maximal weight down-right path (i.e. with steps $(i, j) \rightarrow(i+1, j),(i, j+1))$ in $D=\Phi(\pi)$ from $(1,1)$ to $(n, m)$. This is similar to the classical RSK account, see [Pak01, Sag01]. 


\section{A multivariate identity}

The following notion (related to the previous notion of descent level sets) will be important for us and used throughout. For a plane partition $\pi$, define the descent set

$$
\operatorname{Des}(\pi):=\left\{(i, j): \pi_{i j}>\pi_{i+1 j}\right\} .
$$

Denote also $\operatorname{des}(\pi)=|\operatorname{Des}(\pi)|$.

As a first application of the bijection $\Phi$ described in previous section we prove the following identity which is a source of several formulas given later.

Theorem 4.1. The following formula holds:

$$
\sum_{\pi} \prod_{(i, j) \in \operatorname{Des}(\pi)} x_{i} z_{\pi_{i j}}=\prod_{i=1}^{n} \prod_{\ell=1}^{m} \frac{1}{1-x_{i} z_{\ell}}
$$

where the sum runs over plane partitions $\pi \in \mathrm{PP}(\infty, n, m)$.

Proof. Let us write the generating function

$$
\sum_{D} \prod_{i=1}^{n} \prod_{\ell=1}^{m}\left(x_{i} z_{\ell}\right)^{d_{i \ell}}=\prod_{i=1}^{n} \prod_{\ell=1}^{m} \frac{1}{1-x_{i} z_{\ell}}
$$

over all $\mathbb{N}$-matrices $D=\left(d_{i \ell}\right)$ with $n$ rows and $m$ columns, $1 \leq i \leq m, 1 \leq \ell \leq n$. Applying the bijection $\Phi^{-1}$, the l.h.s of (8) rewrites as follows

$$
\sum_{D} \prod_{i=1}^{n} \prod_{\ell=1}^{m}\left(x_{i} z_{\ell}\right)^{d_{i \ell}}=\sum_{\pi} \prod_{i=1}^{n} \prod_{\ell=1}^{m}\left(x_{i} z_{\ell}\right)^{\left|\left\{j: \pi_{i j}=\ell>\pi_{i+1 j}\right\}\right|}=\sum_{\pi} \prod_{(i, j) \in \operatorname{Des}(\pi)} x_{i} z_{\pi_{i j}},
$$

where the sum is over plane partitions $\pi \in \operatorname{PP}(\infty, n, m)$.

\section{Generalized dual Grothendieck polynomials}

Definition 5.1. Define the polynomials $g_{\lambda}(\mathbf{x} ; \mathbf{z})$ in two sets of variables $\mathbf{x}=\left(x_{1}, \ldots, x_{n}\right)$ and $\mathbf{z}=\left(z_{1}, \ldots, z_{m}\right)$ indexed by partitions $\{\lambda\}$ as follows:

$$
g_{\lambda}(\mathbf{x} ; \mathbf{z}):=\sum_{\operatorname{sh}(\pi)=\lambda} \prod_{(i, j) \in \operatorname{Des}(\pi)} x_{i} z_{\pi_{i j}}
$$

where the sum runs over plane partitions $\pi \in \mathrm{PP}(\infty, n, m)$ of shape $\lambda$. Note that $g_{\lambda}(\mathbf{x} ; \mathbf{z})=0$ unless $\ell(\lambda) \leq n$.

Theorem 5.2. The polynomials $g_{\lambda}(\mathbf{x} ; \mathbf{z})$ satisfy the following properties:

(i) We have the expansion

$$
g_{\lambda}(\mathbf{x} ; \mathbf{z})=\mathbf{x}^{\lambda} s_{\lambda}(\mathbf{z})+\text { lower degree terms }
$$

where $\mathbf{x}^{\lambda}=x_{1}^{\lambda_{1}} \cdots x_{n}^{\lambda_{n}}$. Moreover, each monomial $\mathbf{x}^{\alpha} \mathbf{z}^{\beta}$ in $g_{\lambda}$ has $|\alpha|=|\beta|$.

(ii) We have the formula

$$
g_{\lambda}(\mathbf{x} ; \mathbf{z})=\sum_{\operatorname{sh}(\pi)=\lambda} \mathbf{x}^{d(\pi)} \mathbf{z}^{c(\pi)}, \quad \text { where } \quad \mathbf{x}^{d(\pi)}=\prod_{i=1}^{n} x_{i}^{d_{i}(\pi)}, \quad \mathbf{z}^{c(\pi)}=\prod_{j=1}^{m} z_{j}^{c_{j}(\pi)}
$$


where the sum runs over plane partitions $\pi \in \mathrm{PP}(\infty, n, m)$ of shape $\lambda ; c_{j}(\pi)$ is the number of columns of $\pi$ containing $j$ and $d_{i}(\pi)$ is the number of entries in ith row of $\pi$ that are strictly larger than the entry below.

(iii) $g_{\lambda}\left(1^{n} ; \mathbf{z}\right)=g_{\lambda}(\mathbf{z})$ coincides with the dual Grothendieck polynomial for $\ell(\lambda) \leq n$.

(iv) The following Cauchy-type identity holds

$$
\sum_{\ell(\lambda) \leq n} g_{\lambda}(\mathbf{x} ; \mathbf{z})=\prod_{i=1}^{n} \prod_{j=1}^{m} \frac{1}{1-x_{i} z_{j}}
$$

Proof. (i) A plane partition $\pi$ contributed to the top degree component when the set $\operatorname{Des}(\pi)$ contains all cells of $\pi$, i.e. when $\pi$ is a column-strict plane partition. In this case, the $\mathbf{x}$ monomial will be $\mathbf{x}^{\lambda}$ corresponding to the shape of $\pi$. Summing over such $\pi$, we get the Schur polynomial $s_{\lambda}(\mathbf{z})$. It is clear by definition that monomial expansions have equal $x$ and $z$ degrees.

(ii) The expansion (10) easily follows from the definition and recounting degrees.

(iii) This immediately follows from definition of dual Grothendieck polynomials and the formula (10) when setting $\mathbf{x}=1^{n}$.

(iv) Using the definition (9) and Theorem 4.1 we have

$$
\sum_{\lambda} g_{\lambda}(\mathbf{x} ; \mathbf{z})=\sum_{\pi} \prod_{(i, j) \in \operatorname{Des}(\pi)} x_{i} z_{\pi_{i j}}=\prod_{i=1}^{n} \prod_{j=1}^{m} \frac{1}{1-x_{i} z_{j}}
$$

Corollary 5.3. The following identity holds for dual Grothendieck polynomials

$$
\sum_{\ell(\lambda) \leq n} g_{\lambda}(\mathbf{z})=\prod_{i=1}^{m} \frac{1}{\left(1-z_{i}\right)^{n}}
$$

Proof. Plug $\mathbf{x}=1^{n}$ in (11) and use (iii).

Remark 5.4. Our proof of this identity as well as (11) is essentially bijective, using the map $\Phi$ that preserves weights of $g_{\lambda}$. The identity (12) has an algebraic proof using the family of stable Grothendieck polynomials $\left\{G_{\lambda}\right\}$ dual to $\left\{g_{\lambda}\right\}$ and the Cauchy identity. It uses non-obvious properties of certain automorphisms of $G_{\lambda}$ discussed in [Buc02]. See [Yel19, Yel20].

Remark 5.5. Since $\left\{g_{\lambda}\right\}$ is a deformation of Schur polynomials, the formula (12) can be compared to the following identity for Schur functions [Mac98]

$$
\sum_{\ell(\lambda) \leq n} s_{\lambda}\left(z_{1}, \ldots, z_{n}\right)=\prod_{1 \leq i \leq n} \frac{1}{1-z_{i}} \prod_{1 \leq i<j \leq n} \frac{1}{1-z_{i} z_{j}} .
$$

Remark 5.6. The polynomials $g_{\lambda}(\mathbf{x} ; \mathbf{z})$ are in fact a translated version of the refined dual stable Grothendieck polynomials $\tilde{g}_{\lambda}(\mathbf{t} ; \mathbf{z})$ introduced in [GGL16]. This can be seen via the formula (10); namely, we have $g_{\lambda}(\mathbf{x} ; \mathbf{z})=\mathbf{x}^{\lambda} \tilde{g}_{\lambda}\left(\mathbf{x}^{-1} ; \mathbf{z}\right)$. This implies that $g_{\lambda}(\mathbf{x} ; \mathbf{z})$ is symmetric in the $\mathbf{z}$ variables. 
Problem 5.7. How can the bijection $\Phi$ be extended to prove the Cauchy identity for symmetric Grothendieck polynomials? Namely, the identity

$$
\sum_{\lambda} G_{\lambda}(\mathbf{x}) g_{\lambda}(\mathbf{z})=\prod_{i, j} \frac{1}{1-x_{i} z_{j}} .
$$

Note that our approach works for $\mathbf{x}=1^{n}$ for which we obtained the identity (12). More generally, the problem is about a bijective proof of the skew Cauchy identity proved in [Yel19]

$$
\sum_{\lambda} G_{\lambda / / \mu}(\mathbf{x}) g_{\lambda / \nu}(\mathbf{z})=\prod_{i, j} \frac{1}{1-x_{i} z_{j}} \sum_{\kappa} G_{\nu / / \kappa}(\mathbf{x}) g_{\mu / \kappa}(\mathbf{z})
$$

which would be a generalization of skew RSK for Schur functions given in [SS90].

\section{Two statistics on Plane partitions}

6.1. Up-hooks. For a plane partition $\pi$ and a cell $(i, j)$, define the up-hook

$$
\operatorname{uh}(i, j):=\pi_{i j}+i-1 \text {. }
$$

When $\pi$ presented as a pile of cubes, up-hooks look as follows (here $\mathrm{uh}(i, j)=5+4-1$ ):

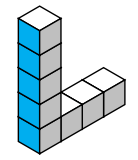

Now, define the up-hook volume of $\pi$ as:

$$
|\pi|_{u h}:=\sum_{(i, j) \in \operatorname{Des}(\pi)} \mathrm{uh}(i, j)
$$

Example 6.1. Consider the plane partition

$$
\pi=\begin{array}{|l|l|l|}
\hline 4 & \mathbf{4} & 2 \\
\hline \mathbf{4} & 2 & \mathbf{2} \\
\hline \mathbf{2} & \mathbf{2} & \multicolumn{2}{|c}{} \\
\cline { 1 - 1 } &
\end{array}
$$

Here the bold entries correspond to $\operatorname{Des}(\pi)$ cells. We have e.g. $\mathrm{uh}(2,1)=4+2-1=5$ and

$$
|\pi|_{u h}=(4+1-1)+(4+2-1)+(2+2-1)+(2+3-1)+(2+3-1)=20 .
$$

Note that $|\pi|=22 \neq|\pi|_{\text {uh }}$.

Theorem 6.2. The following identity holds

$$
\sum_{\pi \in \operatorname{PP}(\infty, n, m)} t^{\operatorname{des}(\pi)} q^{|\pi|_{u h}}=\prod_{i=1}^{m} \prod_{j=1}^{n} \frac{1}{1-t q^{i+j-1}} .
$$

Proof. The identity follows from the multivariate formula in Theorem 4.1 by setting $x_{i}=t q^{i-1}$ and $z_{j}=q^{j}$ which gives

and hence the needed identity.

$$
\prod_{(i, j) \in \operatorname{Des}(\pi)} x_{i} z_{\pi_{i j}}=t^{\operatorname{des}(\pi)} q^{|\pi|_{u h}}
$$

Remark 6.3. Since the proof of Theorem 4.1 is bijective, we have a bijective proof of (13). 
Letting $m, n \rightarrow \infty$ we obtain the following equidistribution of volume and up-hook-volume statistics.

Corollary 6.4 (Equidistribution of volume and up-hook-volume). We have:

$$
\sum_{\pi} q^{|\pi|_{u h}}=\prod_{k=1}^{\infty} \frac{1}{\left(1-q^{k}\right)^{k}}=\sum_{\pi} q^{|\pi|} .
$$

Moreover, the pair statistics $\left(\operatorname{des}(\pi),|\pi|_{u h}\right)$ and $(\operatorname{tr}(\pi),|\pi|)$ are also equidistributed:

$$
\sum_{\pi} t^{\operatorname{des}(\pi)} q^{|\pi|_{u h}}=\prod_{k=1}^{\infty} \frac{1}{\left(1-t q^{k}\right)^{k}}=\sum_{\pi} t^{\operatorname{tr}(\pi)} q^{|\pi|},
$$

where $\operatorname{tr}(\pi)=\sum_{i} \pi_{i i}$ is the trace of a plane partition.

Similarly, for $m \rightarrow \infty$ or $n \rightarrow \infty$ we obtain the following identities.

Corollary 6.5. We have

$$
\begin{aligned}
\sum_{\pi \in \operatorname{PP}(\infty, \infty, m)} q^{|\pi|_{u h}} & =\prod_{j=1}^{\infty}\left(1-q^{j}\right)^{-\min (j, m)} \\
\sum_{\pi \in \operatorname{PP}(\infty, n, \infty)} q^{|\pi|_{u h}} & =\prod_{i=1}^{\infty}\left(1-q^{i}\right)^{-\min (i, n)}
\end{aligned}
$$

Remark 6.6. It would be interesting to see a direct combinatorial argument why the statistics $|\cdot|_{u h}$ and $|\cdot|$ are equidistributed (and when paired with des and trace).

Remark 6.7. The map $\pi_{i j} \mapsto \mathrm{uh}(i, j)=\pi_{i j}+i-1$ for all $i, j$ defines a bijection between (weak) reverse plane partitions (RPP which may contain zero entries) and semistandard Young tableaux (SSYT) (see e.g. [Sta99, Ch. 7.22]). This bijection gives the famous formula

$$
\sum_{\operatorname{sh}(\pi)=\lambda} q^{|\pi|}=q^{|\lambda|-b(\lambda)} s_{\lambda}\left(1, q, q^{2}, \ldots\right)=q^{|\lambda|} \prod_{(i, j) \in \lambda} \frac{1}{1-q^{h_{\lambda}(i, j)}},
$$

where the sum runs over RPP $\pi$ of shape $\lambda, b(\lambda)=\sum(i-1) \lambda_{i}$ and $h_{\lambda}(i, j)=\lambda_{i}-i+\lambda_{j}^{\prime}-j+1$ is the hook length of $(i, j)$ in $\lambda$. A bijective proof of (14) is known as the Hillman-Grassl correspondence [HG76], see also [Sta99, Ch. 7.21-22]. It would be interesting to see if there is any analogous formula when $|\cdot|$ is replaced with the $|\cdot|_{\text {uh }}$ statistic.

6.2. Corner volume. For a plane partition $\pi$, define the corner volume statistic $|\pi|_{c}$ as

$$
|\pi|_{c}:=\sum_{(i, j) \in \operatorname{Des}(\pi)} \pi_{i, j}
$$

Equivalently, $|\pi|_{c}=\sum_{i \geq 1} i c_{i}$, where $c_{i}$ is the number of columns of $\pi$ containing $i$.

Our next result can be viewed as the corner volume counterpart of the classical MacMahon's formula. 
Theorem 6.8. Let $\rho=\left(k^{n}\right)$. The following identities hold:

$$
\begin{aligned}
\sum_{\pi \in \operatorname{PP}(k, n, m)} q^{|\pi|_{c}} & =s_{\rho}\left(1^{n}, q, q^{2}, \ldots, q^{m}\right) \\
\sum_{\pi \in \operatorname{PP}^{\prime}(k, n, m)} q^{|\pi|_{c}} & =s_{\rho}\left(1^{n-1}, q, q^{2}, \ldots, q^{m}\right) \\
\sum_{\pi \in \operatorname{PP}(\infty, n, m)} q^{|\pi|_{c}} & =\prod_{i=1}^{m}\left(1-q^{i}\right)^{-n}
\end{aligned}
$$

One can let $m \rightarrow \infty$ in all these formulas.

For $q=1$ in (15) we recover MacMahon's boxed formula (3); from the second identity (16) we get the formula for the number of plane partitions with the first row length $k$, the first column length $n$, and the first entry at most $m$ :

$$
\left|\mathrm{PP}^{\prime}(k, n, m)\right|=s_{\rho}\left(1^{m+n-1}\right)=|\mathrm{PP}(k, n, m-1)| .
$$

To prove Theorem 6.8 we need some results on dual Grothendieck polynomials.

Lemma 6.9. Let $\rho=\left(k^{n}\right)$. We have

$$
g_{\rho}(\mathbf{z})=s_{\rho}\left(1^{n-1}, \mathbf{z}\right) .
$$

Proof. We show that the Jacobi-Trudi formulas for both polynomials coincide for the shape $\rho$. Note first that by the classical Jacobi-Trudi formula we have

$$
s_{\rho}\left(1^{n-1}, \mathbf{z}\right)=\operatorname{det}\left[e_{n-i+j}\left(1^{n-1}, \mathbf{z}\right)\right]_{1 \leq i, j \leq k} .
$$

For the polynomials $g$, we have the following Jacobi-Trudi identity [Yel17]

$$
g_{\lambda}(\mathbf{z})=\operatorname{det}\left[e_{\lambda_{i}^{\prime}-i+j}\left(1^{\lambda_{i}^{\prime}-1}, \mathbf{z}\right)\right]_{1 \leq i, j \leq \lambda_{1}}
$$

which for $\lambda=\rho$ gives (19).

Remark 6.10. This lemma together with the bijection $\Phi$ is important for various probabilistic applications particularly related to certain corner distributions of lozenge tilings, given in our recent works [Yel19a, Yel20a].

Remark 6.11. Jacobi-Trudi-type formulas for dual Grothendieck polynomials were originally presented in [SZ03]. A (combinatorial) proof of (20) is given in [Yel17]. See also [Kim20, AY20] with more general identities for skew shapes.

Remark 6.12. We would also like to point out that dual Grothendieck polynomials of rectangular shapes appear in solutions of discrete Toda equations, see [IN18].

Corollary 6.13. We have the following formula

$$
\sum_{\lambda \subset\left(k^{n}\right)} g_{\lambda}(\mathbf{z})=s_{\left(k^{n}\right)}\left(1^{n}, \mathbf{z}\right)
$$


Proof. Let $\rho=\left(k^{n}\right)$. By the combinatorial definition of $g_{\lambda}$, we have the following branching formula

$$
g_{\rho}(1, \mathbf{z})=\sum_{\lambda \subset \rho} g_{\lambda}(\mathbf{z})
$$

On the other hand, $g_{\rho}(1, \mathbf{z})=s_{\rho}\left(1^{n}, \mathbf{z}\right)$ by Lemma 6.9 .

Proof of Theorem 6.8. Note that for the principal specialization $z_{i}=q^{i}$ we have

$$
g_{\lambda}\left(q, q^{2}, \ldots\right)=\sum_{\operatorname{sh}(\pi)=\lambda} \prod_{(i, j) \in \operatorname{Des}(\pi)} q^{\pi_{i j}}=\sum_{\operatorname{sh}(\pi)=\lambda} q^{|\pi|_{c}} .
$$

The identities (15), (16) now follow from (21) and (18), respectively. The identity (17) follows from (12).

Remark 6.14. By taking appropriate specializations, we can easily generalize our formulas for restricted partitions with parts from any set of nonnegative integers.

Remark 6.15. It is not difficult to show that both of the presented statistics $|\cdot|_{u h}$ and $|\cdot|_{c}$ are superadditive functions on plane partitions, i.e.

$$
\left|\pi+\pi^{\prime}\right|_{\alpha} \geq|\pi|_{\alpha}+\left|\pi^{\prime}\right|_{\alpha}
$$

where $\alpha$ is $u h$ or $c$. Moreover, $|\cdot|_{c}$ is an antinorm, i.e. besides superadditivity we also have

(i) $|\pi|_{c} \geq 0$ and $|\pi|_{c}=0$ implies $\pi=0$

(ii) $|k \pi|_{c}=k|\pi|_{c}$ for $k \in \mathbb{N}$.

However, the item (ii) fails for $|\cdot|_{u h}$ and instead we have

$$
k|\pi|_{c} \leq|k \pi|_{u h}=|\pi|_{u h}+(k-1)|\pi|_{c} \leq k|\pi|_{u h} .
$$

Note also that we always have $|\pi|_{u h},|\pi| \geq|\pi|_{c}$ and $|\cdot|$ is an additive norm.

Remark 6.16. Enumeration of plane partitions for the usual volume has many product formulas for various symmetry classes, see [Sta86]. It would be interesting to see if there are any analogous generating functions for the weights $|\cdot|_{u h}$ or $|\cdot|_{c}$ over some symmetric plane partitions.

\section{Strict tableaux And LONGeSt increasing SUbSequences of WordS}

Say that a plane partition $\pi$ is a strict tableau if it is filled with entries $[n]:=\{1, \ldots, n\}$ such that each $i \in[n]$ appears in exactly one column of $\pi$. We then say that $[n]$ is a filling of $\pi$. Let $\operatorname{ST}(\lambda, n)$ be the set of strict tableaux of shape $\lambda$ with the filling $[n]$ and $f_{\lambda}(n)=|\mathrm{ST}(\lambda, n)|$. We clearly have $f_{\lambda}(n)=0$ unless $\lambda_{1} \leq n \leq|\lambda|$ and $f_{\lambda}(|\lambda|)=f^{\lambda}$ is the number of standard Young tableaux (SYT) of shape $\lambda$.

Denote also by $W_{n, m}$ the set of words of length $n$ in the alphabet $[m]$.

Theorem 7.1. The following identity holds:

$$
\sum_{\lambda \subset\left(n^{m}\right)} f_{\lambda}(n)=m^{n} .
$$

Furthermore, there is a bijection between the sets $W_{n, m}$ and $\operatorname{ST}(\lambda, n)$ for $\lambda \subset\left(n^{m}\right)$. 
Proof. When we apply the bijection $\Phi$ on a strict tableau $\pi$, the resulting matrix $D$ contains a single 1 in every of its $n$ columns, and has at most $m$ rows. Such $D$ clearly corresponds to a unique word in $W_{n, m}$ by writing down row indices of 1 's in $D$. Conversely, it is easy to see that any word in $W_{n, m}$ converted into a matrix $D$ and applying the inverse map $\Phi^{-1}$ gives a unique element of $\operatorname{ST}(\lambda, n)$ for some $\lambda \subset\left(n^{m}\right)$.

Remark 7.2. As it was noticed by one of the referees, a map from strict tableaux to words has the following simple description: For each $i \in[n]$ write down the largest row index in which $i$ appears.

We now show an algebraic motivation of strict tableaux and a way to derive the formula (22). The numbers $f_{\lambda}(n)$ can be extracted from the so-called Plancherel specialization of the ring of symmetric functions $\Lambda$ applied to the basis $\left\{g_{\lambda}\right\}$. Namely, let $\theta: \Lambda \rightarrow \mathbb{R}[[t]]$ be the specialization (algebra homomorphism) given by

$$
\theta: p_{1} \mapsto t \text { and } \theta: p_{k} \mapsto 0 \text { for } k \geq 2
$$

where $p_{k}=\sum_{i} x_{i}^{k}$ are the power sum symmetric functions which are generators of $\Lambda$.

Proposition 7.3. The following expansion holds

$$
\theta\left(g_{\lambda}\right)=\sum_{n=\lambda_{1}}^{|\lambda|} f_{\lambda}(n) \frac{t^{n}}{n !}
$$

Proof. Follows from the fact that for any symmetric function $g \in \Lambda$ we have:

$$
\theta(g)=\sum_{n}\left[x_{1} \cdots x_{n}\right] g \frac{t^{n}}{n !}
$$

where $\left[x_{1} \cdots x_{n}\right] g$ is the coefficient of $x_{1} \cdots x_{n}$ in the monomial expansion of $g$, see [Ges90, Sta99]. It is easy to see that $\left[x_{1} \cdots x_{n}\right] g_{\lambda}=f_{\lambda}(n)$ hence giving the identity.

Now, using the identity (12) derived earlier, on the other hand we have

$$
\sum_{\ell(\lambda) \leq m} \theta\left(g_{\lambda}\right)=\theta \prod_{i \geq 1}\left(1-x_{i}\right)^{-m}=e^{m t} .
$$

Therefore, combining this with (23) we obtain

$$
\sum_{\ell(\lambda) \leq m} \theta\left(g_{\lambda}\right)=\sum_{\ell(\lambda) \leq m} \sum_{n \geq \lambda_{1}} f_{\lambda}(n) \frac{t^{n}}{n !}=e^{m t}=\sum_{n} m^{n} \frac{t^{n}}{n !}
$$

which by comparing the coefficients of $t^{n}$ proves $(22)$.

Remark 7.4. The identity (22) derived from the Plancherel specialization on dual Grothendieck polynomials is an analog of the classical Frobenius-type identity

$$
\sum_{\lambda \vdash n} f^{\lambda} s_{\lambda}\left(1^{m}\right)=m^{n}
$$

that follows from the Plancherel specialization and $\left(1^{m}\right)$ applied on Schur functions in the Cauchy identity. The bijection $\Phi$ is then an analog of the RSK. 
Remark 7.5. Strict tableaux arise naturally as walks on the so-called $\beta$-filtration of Young's lattice, see [Yel19]. This filtration is an example of more general dual filtered graphs introduced and studied in [PP18].

For a word $w=w_{1} \cdots w_{n} \in W_{n, m}$, a weakly increasing subsequence is a sequence of the form

$$
w_{i_{1}} \leq \cdots \leq w_{i_{k}}, \quad 1 \leq i_{1}<\cdots<i_{k} \leq n,
$$

where $k$ is its length. Let $L_{i}(w)$ be the length of the largest weakly increasing subsequence of $w$ using the letters $\{m-i+1, \ldots, m\}$. In particular, $L_{1}(w)$ is just the number of $m$ 's in $w$ and $L_{m}(w)$ is the length of the longest weakly increasing subsequence of $w$.

For a word $w=w_{1} \cdots w_{n} \in W_{n, m}$ let $D(w)=\left(d_{i j}\right)$ be an $m \times n$ 01-matrix defined as $d_{w_{i}, i}=1$ for all $i \in[n]$ and all other elements of $D$ are zero. Note that $D$ has single 1 in each column.

Theorem 7.6. Let $w \in W_{n, m}$ and $\pi=\Phi^{-1}(D(w))$. Then the shape of $\pi$ is $\left(L_{m}(w), \ldots, L_{1}(w)\right)$.

Proof. Let $\lambda_{1} \geq \cdots \geq \lambda_{m} \geq 0$ be the shape of $\pi$. We need to show that $\lambda_{k}=L_{m-k+1}(w)$ for all $k \in[m]$.

Take any down-right lattice path $\Pi$ from $(k, 1)$ to $(m, n)$. Then it is not difficult to see that the descent level sets $D_{i \ell}$ for $(i, \ell) \in \Pi$ are pairwisely disjoint, note that $i \geq k$. Hence we obtain

$$
\sum_{(i, \ell) \in \Pi} d_{i \ell}=\sum_{(i, \ell)}\left|\left\{j: \pi_{i j}=\ell>\pi_{i+1 j}\right\}\right| \leq \lambda_{k}
$$

On the other hand, suppose the $k$-th row of $\pi$ has entries $\left(\ell_{1} \geq \cdots \geq \ell_{s}\right)$ where $s=\lambda_{k}$. Assume the entries $\ell_{1}, \ldots, \ell_{s}$ end in the rows $i_{1} \geq \cdots \geq i_{s}$ of $\pi$. Then there is a down-right path $\Pi$ from $(k, 1)$ to $(m, n)$ that contains all points $\left(i_{s}, \ell_{s}\right), \ldots,\left(i_{1}, \ell_{1}\right)$. The weight of any such path is at least $\sum_{j} d_{i_{j} \ell_{j}} \geq s=\lambda_{k}$. Combining this with the inequality (24) we obtain that

$$
\lambda_{k}=\max _{\Pi} \sum_{(i, \ell) \in \Pi} d_{i \ell}
$$

where the maximum is taken over down-right paths $\Pi$ from $(k, 1)$ to $(m, n)$. Finally, by translating $D(w)$ to $w$, one can easily see that $L_{m-k+1}(w)$ is also the maximal weight of an down-right path from $(k, 1)$ to $(m, n)$ in $D$.

Example 7.7. Let $w=132434 \in W_{6,4}$. We have

$$
D(w)=\left(\begin{array}{cccccc}
1 & 0 & 0 & 0 & 0 & 0 \\
0 & 0 & 1 & 0 & 0 & 0 \\
0 & 1 & 0 & 0 & 1 & 0 \\
0 & 0 & 0 & 1 & 0 & 1
\end{array}\right) \quad \Phi^{-1}(D(w))=\begin{array}{ll|l|l|l|}
\hline 6 & 5 & 3 & 1 \\
\hline 6 & 5 & 3 \\
\hline 6 & 5 & 2 \\
\hline 6 & 4 &
\end{array} \mid \pi \in \operatorname{ST}(4332,6)
$$

$L_{4}(w)=\lambda_{1}=4$ e.g. the subsequence $(1,2,4,4) ; L_{3}(w)=\lambda_{2}=3$ e.g. the subsequence $(2,4,4) ;$ $L_{2}(w)=\lambda_{3}=3$ e.g. the subsequence $(3,4,4) ; L_{1}(w)=\lambda_{4}=2$ with the subsequence $(4,4)$.

Remark 7.8. This theorem is an analog of Greene's theorem for RSK, see e.g. [Sta99, Ch. 7].

Remark 7.9. Probabilistic versions of these results were discussed in [Yel20a]. 


\section{Monotonicity And Bounds For Descents EnUmeration FunCtions}

For $\alpha=\left(\alpha_{1}, \ldots, \alpha_{m}\right) \in \mathbb{N}^{m}$, let $D_{\alpha}(k, n, m)$ be the number of plane partitions $\pi \in \operatorname{PP}(k, n, m)$ with $\alpha_{i}$ descents of entry $i$ in $\pi$ for $i \in[1, m]$; alternatively entry $i$ is in $\alpha_{i}$ columns of $\pi$.

For $\alpha, \beta \in \mathbb{N}^{m}$ we write $\beta \succeq \alpha$ and say that $\beta$ dominates $\alpha$ if

$$
\sum_{i=1}^{\ell} \beta_{i} \geq \sum_{i=1}^{\ell} \alpha_{i} \quad \text { for all } \ell \in[1, m] .
$$

Theorem 8.1. The following properties hold:

(i) symmetry: $D_{\alpha}(k, n, m)$ does not change under permutations of $\left(\alpha_{1}, \ldots, \alpha_{m}\right)$

(ii) monotonicity:

$$
D_{\alpha}(k, n, m) \geq D_{\beta}(k, n, m) \text { for } \beta \succeq \alpha \text { and }|\alpha|=|\beta| .
$$

Proof. By the definition of $g_{\lambda}$ and Lemma 6.9, we have for $\rho=\left(k^{n}\right)$

$$
\sum_{\alpha \in \mathbb{N}^{m}} D_{\alpha}(k, n, m) x^{\alpha}=g_{\rho}\left(1, x_{1}, \ldots, x_{m}\right)=s_{\rho}\left(1^{n}, x_{1}, \ldots, x_{m}\right) .
$$

In particular, (i) becomes evident since $g_{\rho}$ is a symmetric polynomial. Let us expand the Schur polynomial using the branching formula

$$
s_{\rho}\left(1^{n}, x_{1}, \ldots, x_{m}\right)=\sum_{\lambda \subset \rho} s_{\lambda}\left(x_{1}, \ldots, x_{m}\right) s_{\rho / \lambda}\left(1^{n}\right)=\sum_{\lambda \subset \rho} \sum_{\alpha} K_{\lambda \alpha} x^{\alpha} s_{\rho / \lambda}\left(1^{n}\right),
$$

where $K_{\lambda \alpha}$ is the Kostka number (i.e. the coefficients in the monomial expansion of $s_{\lambda}$, or the number of SSYT of shape $\lambda$ of type $\alpha$ ). Combining these identities we obtain the following formula

$$
D_{\alpha}(k, n, m)=\sum_{\lambda \subset \rho} K_{\lambda \alpha} s_{\rho / \lambda}\left(1^{n}\right) .
$$

The item (ii) now follows from a similar inequality for Kostka numbers [Mac98], $K_{\lambda \alpha} \geq K_{\lambda \beta}$ for $\beta \succeq \alpha$. Indeed we get

$$
D_{\alpha}(k, n, m)=\sum_{\lambda \subset \rho} K_{\lambda \alpha} s_{\rho / \lambda}\left(1^{n}\right) \geq \sum_{\lambda \subset \rho} K_{\lambda \beta} s_{\rho / \lambda}\left(1^{n}\right)=D_{\beta}(k, n, m)
$$

as needed.

Corollary 8.2. Let $\alpha \in \mathbb{N}^{m}$ with $|\alpha|=N \leq \min (k, m)$. The following inequalities hold

$$
\left(\begin{array}{c}
n+N \\
N
\end{array}\right) \leq D_{\alpha}(k, n, m) \leq n^{N}
$$

Proof. From the monotonicity (ii) we have

$$
D_{(N, 0, \ldots, 0)}(k, n, m) \leq D_{\alpha}(k, n, m) \leq D_{\left(1^{N}, 0, \ldots, 0\right)}(k, n, m) .
$$

Recall that $D_{(N, 0, \ldots, 0)}(k, n, m)$ is the number of plane partitions with a single entry occupying $N \leq k$ columns in the rectangle $\left(k^{n}\right)$. This number is clearly $\left(\begin{array}{c}n+N \\ N\end{array}\right)$ which gives the lower bound.

Next, note that $D_{\left(1^{N}, 0, \ldots, 0\right)}(k, n, m)$ is the number of plane partitions with entries $\{1, \ldots, N\}$ such that each entry occupies a single column. Note that each entry is uniquely determined by its lowest row position which can be any number from 1 to $n$. Hence the total number of such plane partitions is at most $n^{N}$ which gives the upper bound. 
Note that the bounds do not depend on $k, m$. The lower bound is sharp and the inequalities imply sharp asymptotics e.g. for $k, m \geq N$ and constant $n$

$$
\log D_{\alpha}(k, n, m)=N \log n+o(N) \text { as } N \rightarrow \infty .
$$

Otherwise, e.g. for $N=\Theta(n)$ we get the bounds

$$
\Theta(N) \leq \log D_{\alpha}(k, n, m) \leq N \log n .
$$

Note also that there is another upper bound (the formula when $k \rightarrow \infty$ is unbounded is not difficult to derive using our bijection)

$$
D_{\alpha}(k, n, m) \leq D_{\alpha}(\infty, n, m)=\prod_{i=1}^{m}\left(\begin{array}{c}
n+\alpha_{i}-1 \\
\alpha_{i}
\end{array}\right) \leq\left(\begin{array}{c}
m n+N-1 \\
N
\end{array}\right) .
$$

\section{ACKNOWLEDGEMENTS}

I am grateful to Askar Dzhumadil'daev, Igor Pak, and Pavlo Pylyavskyy for many helpful conversations. I am also grateful to the referees for careful reading of the text and many useful comments.

\section{REFERENCES}

[AY20] A. Amanov and D. Yeliussizov, Determinantal formulas for dual Grothendieck polynomials, arXiv:2003.03907, 2020.

[Buc02] A. Buch, A Littlewood Richardson rule for the K-theory of Grassmannians, Acta Math. 189 (2002), 37-78.

[GGL16] P. Galashin, D. Grinberg, G. Liu, Refined dual stable Grothendieck polynomials and generalized BenderKnuth involutions, Electronic J. Combin. 23 (2016): 3-14.

[Ges90] I. Gessel, Symmetric functions and P-recursiveness, J. Combin. Theory Ser. A 53 (1990), $257-285$.

[HG76] A. Hillman and R. Grassl, Reverse plane partitions and tableau hook numbers, J. Combin. Theory Ser. A 21 (1976), 216-221.

[IN18] S. Iwao and H. Nagai, The discrete Toda equation revisited: dual $\beta$-Grothendieck polynomials, ultradiscretization, and static solitons, Journal of Physics A: Mathematical and Theoretical 51 (2018): 134002 .

[LP07] T. Lam and P. Pylyavskyy, Combinatorial Hopf algebras and K-homology of Grassmannians, Int. Math. Res. Not. Vol. 2007, (2007), rnm 125.

[Mac98] I. G. Macdonald, Symmetric functions and Hall-Littlewood polynomials, Oxford Univ. Press, Oxford (1998).

[Mac16] P. A. MacMahon, Combinatory Analysis, vols.1 and 2, Cambridge University Press, 1916.

[Pak01] I. Pak, Hook length formula and geometric combinatorics, Sém. Lothar. Combin. 46 (2001): 6.

[PP18] R. Patrias and P. Pylyavskyy, Dual filtered graphs, Algebraic Combinatorics 1.4 (2018), 441-500.

[Kim20] J. S. Kim, Jacobi-Trudi formula for refined dual stable Grothendieck polynomials, arXiv:2003.00540, 2020

[Kra16] C. Krattenthaler, Plane partitions in the work of Richard Stanley and his school, The mathematical legacy of Richard P. Stanley (2016), 246-277.

[Sag01] B. Sagan, The Symmetric Group, Springer, New York, 2001.

[SS90] B. Sagan, R. Stanley, Robinson-Schensted algorithms for skew tableaux, J. Combin. Theory Ser. A 55 (1990), 161-193.

[SZ03] M. Shimozono and M. Zabrocki, Stable Grothendieck symmetric functions and $\Omega$-calculus, preprint, 2003 .

[Sta99] R. Stanley, Enumerative Combinatorics, Vol. 2, Cambridge, 1999. 
[Sta86] R. Stanley, Symmetries of plane partitions, J. Combin. Theory Ser. A 43 (1986), 103-113.

[Yel17] D. Yeliussizov, Duality and deformations of stable Grothendieck polynomials, J. Algebraic Combin. 45 (2017), 295-344.

[Yel19] D. Yeliussizov, Symmetric Grothendieck polynomials, skew Cauchy identities, and dual filtered Young graphs, J. Combin. Theory Ser. A 161 (2019), 453-485.

[Yel19a] D. Yeliussizov, Random plane partitions and corner distributions, arXiv:1910.13378, 2019.

[Yel20] D. Yeliussizov, Positive specializations of symmetric Grothendieck polynomials, Adv. Math. 363 (2020), Article 107000, 35 p.

[Yel20a] D. Yeliussizov, Dual Grothendieck polynomials via last-passage percolation, C. R. Math. Acad. Sci. Paris 358 (2020), 497-503.

KBTU, Almaty, Kazakhstan

Email address: yeldamir@gmail.com 